ROCZNIKI HUMANISTYCZNE

Tom LXVIII, zeszyt $12-2020$

DOI: https://doi.org/10.18290/rh206812-10

RICHARD BEYER

\title{
KONSTRUKTIVISMUS UND INTUITION. EINE ANALYSE VON BÉLA BARTÓKS KLAVIERSTÜCK SUBJECT AND REFLECTION AUS DEM MIKROKOSMOS
}

$\mathrm{Zu}$ den Charakteristika der Neuen Musik des 20. Jahrhunderts gehört die Veränderbarkeit sämtlicher musikalischer Parameter bis hin zur Erweiterung der Musikauffassung selbst: Alles kommt in Fluss. Neue Erfahrungen und neues Denken in Wissenschaft, Kunst und Philosophie führen zu einem heterogenen, dem Zeitgeist entsprechenden Stilpluralismus. Die Verfügungsmöglichkeit über ein vielfältiges, zeitlich und räumlich extrem differenziertes musikalisches Repertoire mit all seinen inneren Widersprüchen und Tendenzen zur Dissoziation erweist sich als Problem. So schwanken die bedeutenden kompositorischen Entwürfe des Jahrhunderts generell betrachtet zwischen Freiheit und unterschiedlich strengen Arten musikalischer Materialordnung.

Béla Bartók (1881-1945) ist stilistisch seinen eigenen Weg gegangen. Dieser wurde nicht wie etwa im Falle Schönbergs von einer Schule aufgegriffen, gehört aber u.a. gerade wegen seiner Individualität und der kreativen Synthese von Geist und Form traditioneller europäischer Kunstmusik, zeitgenössischer Meister wie Debussy, Strawinsky und Schönberg sowie folkloristischer Elemente, die sich sowohl konkret als auch eher vom Gestus her ausprägen, zu den vielbeachteten Lösungen des 20. Jahrhunderts. Bartók begründete seine musikalischen Innovationen mit dem Verweis auf ähnlich vitale melodische, rhythmische oder harmonische Phänomene in der authentischen Volks- oder genauer Bauernmusik: Liedern und Tänzen, die er wie alles tief im Landleben Verwurzelte als naturhaft, unverfälscht begriff und deswegen überaus

Prof. Dr. RichARD BEYER - Hochschule für katholische Kirchenmusik und Musikpädagogik, Regensburg; e-mail: richard-beyer@t-online.de.

Prof. RichARD BEYER - The College of Catholic Church Music and Musical Education in Regensburg, Germany; e-mail address: richard-beyer@t-online.de. 
schätzte. Auch das nationale, ungarische Moment spielte eine wichtige Rolle. Voller Inspiration und Spontaneität strebte er danach, aus Altem Neues zu schaffen. Über technische Details seiner Werke äußerte er sich nur ungern, weil er der Auffassung war, bei Diskussionen die Kunst betreffend sei dies unnatürlich und die Musik solle für sich selbst sprechen. Analysen der Kompositionstechnik insbesondere der von Mitte der zwanziger Jahre bis 1937 geschriebenen Werke - eine Phase, die in der Bartók-Forschung als klassische mittlere Periode bezeichnet wird (Somfai 394) - zeigen aber auch einen sehr deutlich ausgeprägten Strukturalismus. In diese Zeit fällt der Mikrokosmos. Bartók selbst berichtete in einem Zeitungsartikel vom 3. Oktober 1940 über die allmähliche Entstehung dieses Werkes in den Jahren 1926 und 1932-1939:

\begin{abstract}
Ein Stück des Mikrokosmos war ursprünglich das zehnte Stück der Neun kleinen Klavierstücke, die schon im Jahre 1926 erschienen und bei denen es aus irgendeinem Grunde weggelassen wurde. Schon damals beschäftigte mich der Gedanke, leichte Klaviermusik für den Anfangsunterricht zu schreiben. Eigentlich machte ich mich erst im Sommer des Jahres 1932 an die Arbeit. Damals kamen ungefähr 40 Stücke zustande, 1933-1934 wiederum an die 40 und in den nächsten Jahren ungefähr 20, bis endlich im Jahr 1938 hundert und einige Stücke fertig waren. Es waren aber immer noch Mängel da. Diese ergänzte ich im vorigen Jahr. So stellte ich z.B. die erste Hälfte des I. Heftes fertig. Zur praktischen Prüfung des Materials ergab sich bald eine ausgezeichnete Gelegenheit. 1933 bat mich mein jüngerer Sohn Péter sehr, dass wir ihn Klavier lernen lassen sollten. Da fasste ich einen kühnen Entschluss und machte mich selbst an diese, mir ein wenig ungewohnte Arbeit. Außer den Sing- und technischen Übungen bekam das Kind nur Mikrokosmos-Musik; ich hoffe, dass sie ihm nützlich war, ich muss aber bekennen, dass ich selbst viel aus diesen Experimenten lernte (...). (Lesznai $164 \mathrm{f}$ )
\end{abstract}

Die sechs Bände des Mikrokosmos enthalten 153 Klavierstücke in progressiver Schwierigkeit, von den ersten Anfängen bis zur pianistischen Reife. Die Kompositionen sind in der Synthese der eingangs genannten Einflussbereiche, die stärkste Impulse für Ausdruck und Wesen von Bartóks Musik gaben, durchaus repräsentativ für sein Lebenswerk. Gleichzeitig spiegeln sie seine vier Tätigkeitsfelder als Pianist, Komponist, Pädagoge und Musikforscher: Stil und Anlage der Stücke sind pianistisch, dabei handelt es sich aber nicht um abstrakte klaviertechnische Übungen, sondern um geistig und musikalisch durchaus anspruchsvolle Ideen mit künstlerischer Gültigkeit, die der innovativen Phantasie und Kreativität des Komponisten entspringen. Der Aufbau des Gesamtwerkes erfolgt methodisch und systematisch zu pädagogischen Zwecken: Musikalische Bildung entwickelt sich durch die Einführung in Neue 
Musik auf verschiedenen Stufen der Spielbarkeit. Schließlich resultiert die Integration der von westlichem Einfluss unberührten Folklore verschiedener Völker des Balkans aus Bartóks musikethnologischen Studien.

Der Mikrokosmos demonstriert gerade durch die modellhafte Überschaubarkeit der Stücke quasi als klingende Kompositionslehre in einzigartiger Weise Bartóks Kompositionstechnik. Damit ähnelt das Werk in gewisser Weise den Inventionen Johann Sebastian Bachs, der 1723 im Vorwort der Reinschrift den Zweck dieser Stücke umreißt: „,...) gute inventiones nicht alleine zu bekommen, sondern auch selbige wohl durchzuführen, am allermeisten aber eine cantable Art im Spielen zu erlangen und darneben einen starcken Vorschmack von der Composition zu überkommen".

Der Titel Mikrokosmos hat doppelte Bedeutung: Eine Klavierschule zumindest in den ersten Bänden - als Welt für Kleinere, aber auch als Welt, die in verkleinerter Form das (musikalische) Universum abbildet. Diese weitreichenden philosophischen Implikationen gehen auf ein seiner Zeit in Ungarn recht bekanntes, 1856-1864 entstandenes Buch des deutschen Philosophen Rudolf Hermann Lotze (1817-1881) zurück, der übrigens einer von Hugo Riemanns akademischen Lehrern war. Als Reaktion auf den Aufstieg der Naturwissenschaften im Industriezeitalter versuchte Lotze in seiner Philosophie eine Synthese zwischen der metaphysischen Tradition des deutschen Idealismus und der modernen Wissenschaft auf empirischer Basis induktiv zu begründen. Sein System des Idealismus basiert auf der Ethik und der Wirklichkeit des Bewusstseins. Dabei unterscheidet er das, was nach naturwissenschaftlichen Kriterien ist, die Wirklichkeit, von dem, was aufgrund von Wertbegriffen gilt, ohne wahrhaft sein zu müssen. Die Gesetze der Wirklichkeit sind nur Bedingungen zur Verwirklichung des Guten. Die Fülle der Einzelerscheinungen verbindet sich im Reich der Werte - dieser Begriff wurde von ihm in die Ethik eingeführt - zum Guten. Ein ähnlich einheitliches, umfassendes Bewusstsein von Mensch, Welt und Universum findet sich in der mittelalterlichen Philosophie, nach deren Auffassung die Vielgestaltigkeit des Individuellen die Schöpfungsordnung des Kosmos insgesamt wiederspiegelt.

Die quantitativ und qualitativ gewichtigste Gruppe von Stücken im Mikrokosmos kreist um musikalische Phänomene bzw. Kompositionstechniken. Wie bereits der Titel verrät, gehört hierzu auch Subject and Reflection (ungarisch Tükrözödés), Band VI, Nr.141, eine überschaubar dimensionierte Etüde in sparsamer Schreibweise für gegenläufige, teils rhythmisch selbständige Bewegungen beider Hände. An zwei Stellen muss gekreuzt werden (Takt 76 und 78). 
Elementare Polyphonie jeweils innerhalb einer Hand ergibt sich durch Kombination von bewegter und liegender Stimme. Als Artikulationsmöglichkeiten werden überwiegend Legato, gelegentlich Portato geübt. Typisch für die Kompositionen des Mikrokosmos ist Bartóks genaue Zeitangabe am Ende (1 min. $16 \mathrm{sec}$.). Sie zeigt die besondere Bedeutung des richtigen Tempos. Häufige Taktwechsel, auch mit zusammengesetzten Taktarten, vor allem in der mehrfach wiederkehrenden Motivgruppe des Anfangs, rufen ebenso den Eindruck imaginärer Folklore hervor wie die meist einfache Rhythmik und die kurzen, mit vielen Motivwiederholungen kreisenden melodischen Bögen. Die im Titel angedeutete kontrapunktische Satztechnik der Horizontalspiegelung zweier Stimmen (bzw. die Gegenbewegung beider Hände) wird das ganze Stück hindurch angewendet. Dabei markieren Orgelpunkte in der Satzmitte bzw. genauer gesagt liegende Stimmen die wechselnden Spiegelachsen. Die strenge Linearität der Außenstimmen zunächst in völlig gleicher, ab Takt 40 in überwiegend komplementärer Rhythmik führt bisweilen zu unkonventionellen Klängen mit unterschiedlichem Dissonanzgrad.

Die formale Anlage ist rondoartig. Der Refrain (Takte 1-14, Notenbeispiel 1) besteht aus der siebentaktigen Motivgruppe des Anfangs - eine unschematische Alternative zur traditionellen, auf Zweierpotenzen beruhenden Taktgruppenstruktur, die sogleich geringfügig abgewandelt, beinahe wie ein Echo wiederholt wird. Die Töne des B-Dur-Dreiklangs sind durch rhythmische Dehnung als Melodiegerüst der Oberstimme hervorgehoben. Unisoni kennzeichnen Ruhe- bzw. Zielpunkte. Der Refrain wird wieder aufgenommen in den Takten 23-29, 40-46 und 63-73, zuletzt nur noch Motive daraus im steigernden Schlussteil ab Takt 74. Eine Art Variierungsverfahren gewährleistet Spannungsverdichtung durch zunehmende rhythmische Verschiebungen, die schließlich quasi kanonische Stimmführungen verursachen. In den Takten 63-69 erreicht die Polyphonie ihre größte rhythmischmelodische Komplexität. 

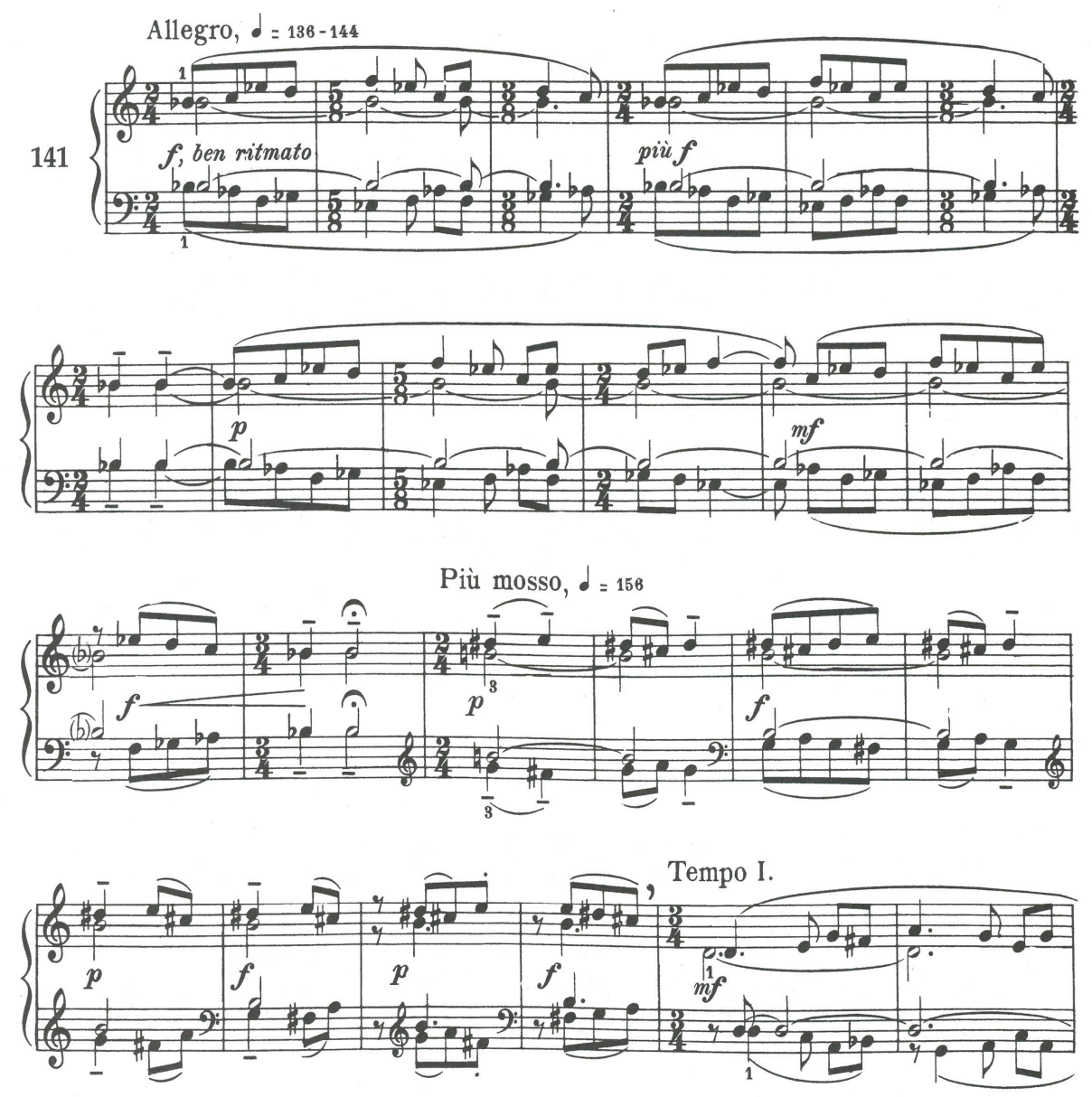

Notenbeispel 1. B. Bartók, Subject and Reflection (Tükrözödés), T. 1-24

Drei Couplets alternieren dynamisch gegensätzlich mit dem Refrain, zu dem sie jeweils unterschiedliche Motivverwandtschaft besitzen. Das erste Couplet (Takte 15-22, Notenbeispiel 1) nimmt die Kreisbewegung des Refrains wieder auf, jedoch verengt von Quint- auf Terzambitus und mit dis statt $d$ als mittlerem Ton des Zirkels. Abgesehen vom Piano-/Forte-Kontrast fällt klanglich eine gewisse Dominanz des übermäßigen Dreiklangs auf. Im zweiten Couplet (Takte 30-39) führen Akzente zu immanenten metrischen Verschiebungen. Es bringt die Töne der Refrainmelodie in freier Umstellung, ebenso wie das dritte Couplet (Takte 47-62, Notenbeispiel 2), das außerdem 
die latenten Quartbeziehungen der Refrainoberstimme offen zu Tage treten lässt und dadurch einen etwas anderen Gestus besitzt. Vergleichbar dem Refrain verursacht hier die Verschiebung um einen Achtelwert rhythmisch komplementäre, kanonartige Impulse. Auch der Ambitus hat Ende des dritten Couplets seine größte Ausdehnung: War der Quintumfang bereits im zweiten Couplet wieder erreicht, so spreizt er sich nun zum extrem weiten, fünfoktavigen Klangraum, der schwungvoll mit Oktavsprüngen geräuschhafter Großsekunddissonanzen durchmessen wird. Letztere gewinnt Bartók durch Motivabspaltung, denn außer im dritten Couplet spielten sie zuvor schon im zweiten eine wichtige Rolle. Geschärft zu kleinen Sekunden werden sie im Schlussteil ab Takt 74 nochmals pointiert aufgegriffen, genau wie das Prinzip der Räumigkeit fünf verschiedener Oktavlagen.
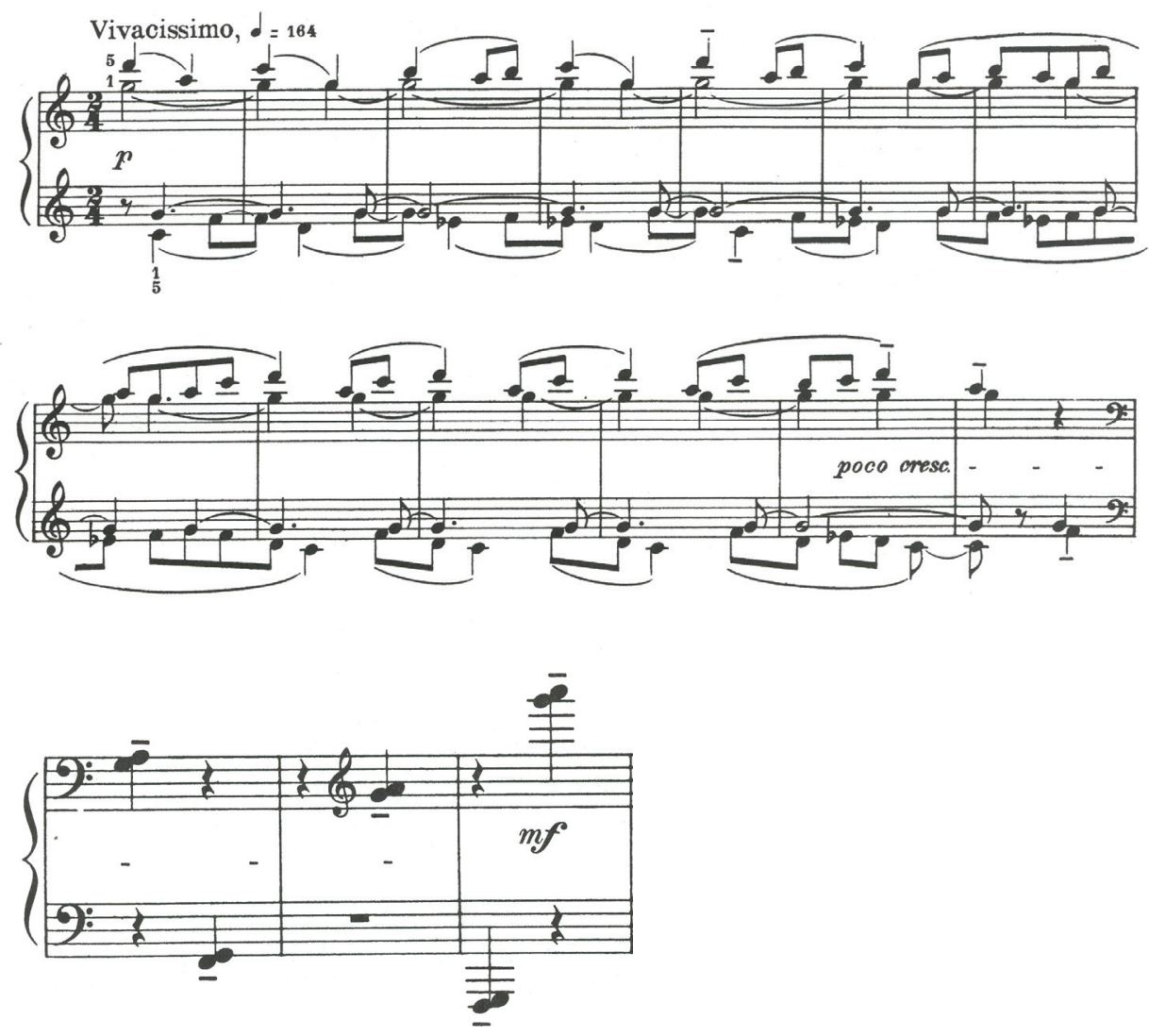

Notenbeispel 2. B. Bartók, Subject and Reflection (Tükrözödés), T. 47-62 
Die Harmonik des Stückes ist streng organisiert und formstiftend. Der jeweilige Spiegelachsenorgelpunkt wirkt stets als Grund- bzw. Zentralton. In den Takten 1-14 nimmt der Ton $b$ diese Bedeutung an. Rechts erklingt ein B-Dur-Pentachord, links ein es-Moll-Pentachord. Der Grundton des Durpentachordes und die Quinte des Mollpentachordes sind oktavidentisch und bilden den Orgelpunkt, doch bewirkt die Spiegelung keine konstruktive Bitonalität bzw. Tonartenmischung von es-Moll und B-Dur, vielmehr liegt eine Tonalitätsform zugrunde, die der ungarische Musikforscher Ernö Lendvai in verschiedenen Veröffentlichungen seit 1947 akustische Tonalität genannt und als besonders charakteristisch für Bartóks Musik herausgestellt hat (Szabolcsi 105ff). Skalar betrachtet handelt es sich um eine den Tönen 8-14 der Obertonreihe entsprechende Heptatonik mit drei geringfügigen Abweichungen aufgrund der gleichschwebenden Temperatur (Ton 11, 13, 14, Notenbeispiel 3).

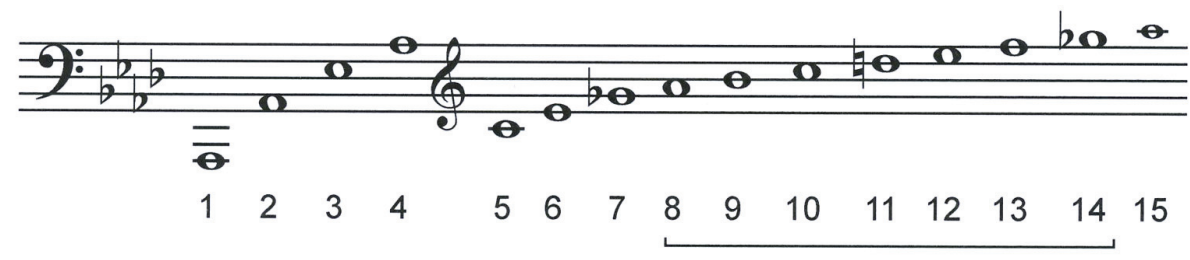

Notenbeispel 3. Partialtonreihe auf As und akustische Skala

Wie Zofia Lissa (Lissa) bezeichnet auch Carl Dahlhaus analoge Tonhöhenordnungen in Melodik und Harmonik Alexander Skrjabins als Klangzentrentechnik (Dahlhaus 322 ff): Klangzentren sind aus dem Tredezimakkord entstandene, emanzipierte Dominantformen, die tonikale Wirkung bekommen, da sie keinem Auflösungszwang mehr unterliegen. Allerdings bietet sich der Terminus akustische Tonalität - als eine von verschiedenen Möglichkeiten - schon deswegen an, weil mehrere bedeutende Komponisten diese Tonhöhenordnung aufgrund ihrer Ähnlichkeit mit dem obengenannten Ausschnitt des Partialtonspektrums im Sinne eines Natursymbols verwenden. Skrjabin beispielsweise begriff seinen mystischen Akkord aus der Symphonischen Dichtung Prometheus, den er selbst - theosophisch spekulierend - für allumfassend und besonders wichtig hielt, als Widerspiegelung der Obertöne 8-14 (Dahlhaus 516); (Der sechstönige Klang entspricht einer quintlosen 
akustischen Skala mit Tritonus im Bass und einer verminderten, einer übermäßigen sowie zwei reinen Quarten darüber). Die Natursemantik findet sich auch in Claude Debussys Schaffen, etwa zu Anfang von La mer. Bartók lernte die akustische Tonalität sowohl in rumänischer und arabischer Bauernmusik kennen, die er - wie bereits dargelegt - für naturhaft hielt, als auch in Werken Liszts, Debussys und Ravels. Obwohl er ähnlich wie Strawinsky eher einer theoretischen Deutung der akustischen Skala als polymodales Phänomen zuneigte, d.h. die ambivalente Klangwirkung hervorhob, die sich aus der Kombination zweier diatonischer Segmente, eines unteren Tetrachordes (bzw. Pentachordes) mit lydischer übermäßiger Quarte und eines oberen Tetrachordes mit mixolydischer kleiner Septime innerhalb desselben Oktavraumes ergibt, evoziert er dennoch in seinen Kompositionen durch diese Tonalitätsform häufig Naturkolorit, so zum Beispiel am Beginn des Tanzspiels Der holzgeschnitzte Prinz, am Schluss der Cantata profana (Die Zauberhirsche) oder im Chor Tavasz (Frühlingslied).

Die vom Höreindruck her schärfere Klanglichkeit der akustischen Skala gegenüber der ebenfalls siebenstufigen Diatonik lässt sich am Quintenband veranschaulichen, einer Darstellungsform des Tonmaterials, die letztlich auf den mittelalterlichen Quintenturm zurückgeht, die Töne aber nicht vertikal, sondern horizontal quintig anordnet. Während die Diatonik eine lückenlose Reihe von sechs Quinten bildet, zeigt die akustische Skala eine größere Spreizung: Acht Quinten mit zwei symmetrisch gruppierten Lücken. Die geringere Anziehungskraft des Grundtons in der akustischen Skala erklärt sich außerdem durch den höheren Tritonusgehalt als zusätzlichem Indikator für den Spannungsgrad. Das diatonische Tonmaterial enthält nur einen Tritonus als Rahmen des Quintenbandes. Genau hier liegen die Lücken der akustischen Skala, die stattdessen am jeweils entgegengesetzten Ende der Quintenreihe die alterierten Varianten der fehlenden Töne einbezieht und daher zwei ineinandergreifende Tritoni jeweils an den Außenpositionen des Quintenbandes aufweist (Notenbeispiel 4); (Gárdonyi 145f). 

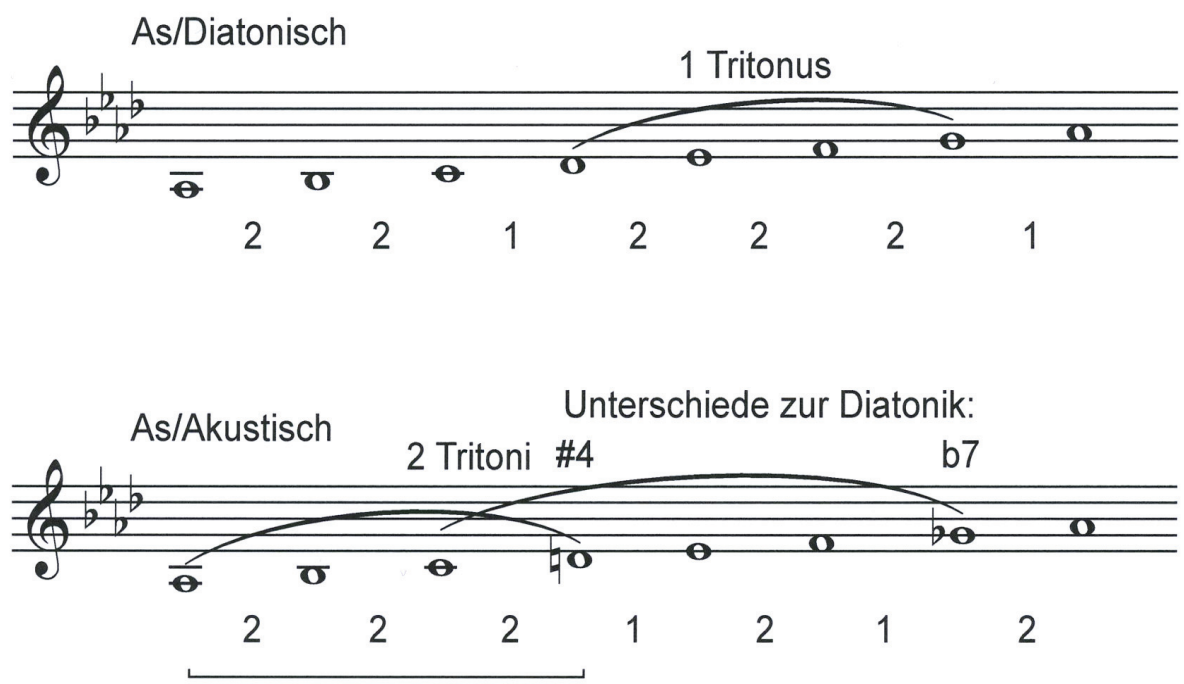

Segment der Ganztonskala

Segment der Ganzton/Halbton-Skala

Diatonische und akustische Skala als Quintenband
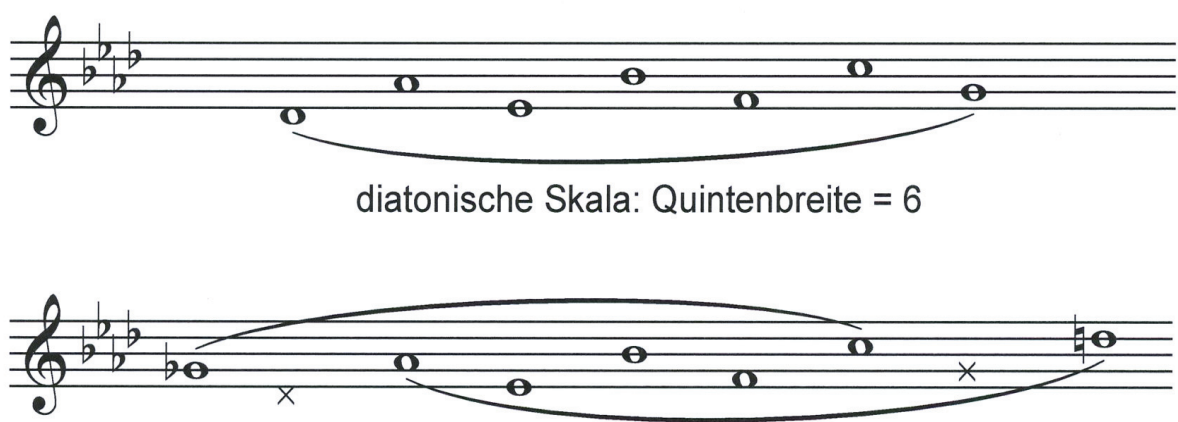

akustische Skala: Quintenbreite $=8$ (einschließlich 2 Lücken)

(Bögen bezeichnen Tritonusrelationen) 
Analog dem diatonischen System kann jeder Ton des Materialvorrats, dem die akustische Skala angehört, Grund- bzw. Zentraltonbedeutung annehmen, so dass wiederum sieben verschiedene Oktavgattungen (Tonleitern bzw. Modi) möglich sind (Gárdonyi 145f). Für diesen Tonvorrat hat der ungarische Musiktheoretiker Lajos Bárdos den Terminus Heptatonia secunda vorgeschlagen (Bárdos Modális; Harmine; Die volksmusikalischen Tonleitern bei Liszt 195ff) um eine erste Form der Siebenstufigkeit, nämlich die diatonische, von einer zweiten, der akustischen, abzugrenzen. Die Benennung der einzelnen Modi der Heptatonia secunda ist in den verschiedenen Ländern und Sprachräumen nicht einheitlich. Sie stammt wesentlich aus der Jazz-Harmonielehre und erfolgt meist nach dem Prinzip der Halbtonverwandtschaft. Dabei werden zusätzlich zum Skalennamen diejenigen Töne angegeben, die von einer diatonischen Leiter auf dem gleichen Grundton halbtönig nach oben oder unten abweichen. Das Standardlehrwerk des amerikanischen Komponisten und Theoretikers Vincent Persichetti enthält einige der heute in der Jazz-Harmonik gebräuchlichen Bezeichnungen für Skalen auf den verschiedenen Stufen der heptatonia secunda (Persichetti): Lydian flat seven oder Mixo \#11 als Synonyme für die akustische Skala. Mixo b6, Locrian \#2, Altered bzw. Super Locrian, MMA = Melodisch Moll aufwärts, Phrygodorian, Lydian augmented (Notenbeispiel 5); (Haunschild 90ff) ${ }^{1}$.

\footnotetext{
${ }^{1}$ Haunschild erläutert einige der Bezeichnungen und gibt noch weitere, etwa Diminished wholetone für Altered.
} 

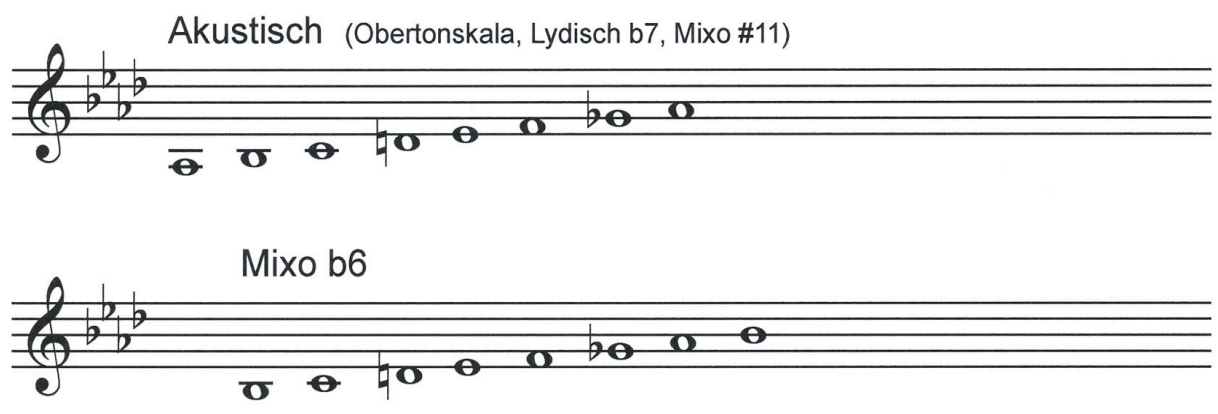

Lokrisch \#2 (Lokrisch 9, Aeolisch b5)
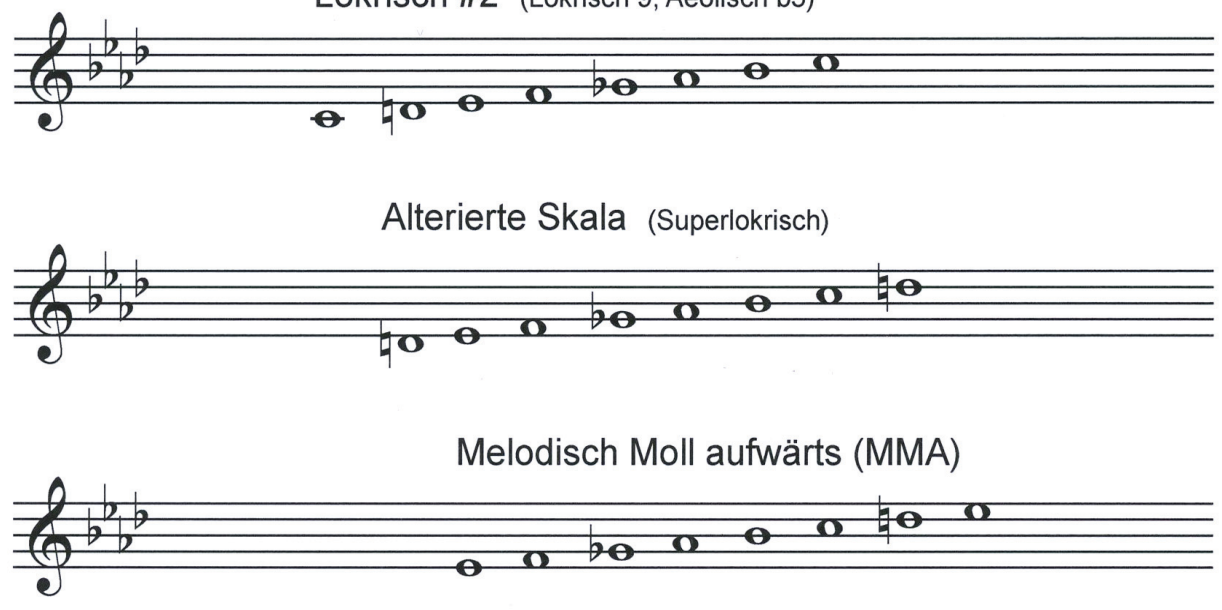

Phrygodorisch (Dorisch b2, Dorisch b9)

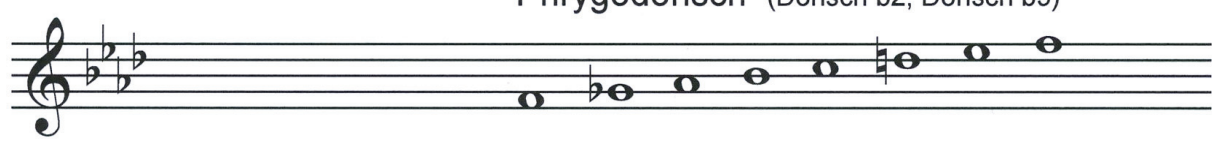

Lydisch übermäßig (Lydisch \#5)

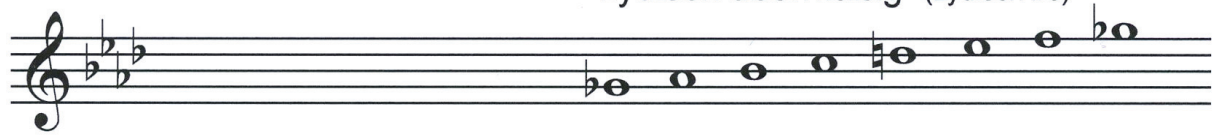

Notenbeispel 5. Das System der heptatonia secunda (nach Bárdos, Budapest 1961, Persichetti, London 1997, Gárdonyi/Nordhoff, Wolfenbüttel 2002) 
Im Jazz wird vielfach melodisch Moll als Matrixleiter des Systems betrachtet. Ausgehend von der genannten Skalenterminologie erweist sich die Tonalität der Takte 1-14 als Mixo b6 - Skala mit Zentralton $b$ innerhalb einer nach as transponierten Heptatonia secunda. Dass Bartók gerade Mixo b6 als Skalenform wählte, könnte mit der Beliebtheit dieses Modus in der Volksmusik zusammenhängen. Nicht umsonst wird gelegentlich äquivok der Terminus Hindu-Scale gebraucht (Gárdonyi, Nordhoff 146), um den Bezug der Leiter zu bestimmten Arten fernöstlicher Folklore zu verdeutlichen. In Westeuropa wird Mixo b6 Melodisches Dur oder Picardisches Moll bzw. Picardisches Aeolisch genannt. Auf dieser Skala basieren viele Lieder der Kurutzen, ungarischer Soldaten, die im Rákóczi-Aufstand (Ende 17., Anfang 18. Jahrhundert) für die Selbständigkeit ihrer Heimat kämpften. Daher stammt der Name Kurutzen-Skala. Bartóks umfangreiche Publikation Das ungarische Volkslied enthält Beispiele solcher Melodien (Bartók 304). Liszt komponierte ebenfalls mit dieser Skala, etwa in der Ungarischen Rhapsodie Nr.6 (Bárdos 195 ff).

Bartók synthetisiert den akustischen Tonvorrat aus fünf Ganzton- und zwei Halbtonschritten kunstvoll im Rahmen eines Nonen- (Doppelquint-) ambitus und zwar in der einzig möglichen Konstellation, die Polarität zweier melodisch und harmonisch Zusammenhang stiftender diatonischer Quinträume erlaubt: Takt 15-22 H-Dur gegen e-Moll (Grundton fehlt) mit Orgelpunkt $h$, Takt 23-29 D-Dur gegen g-Moll mit Orgelpunkt $d$, Takt 30-39 EsDur gegen as-Moll mit Orgelpunkt es, Takt 40-46 Fis-Dur gegen h-Moll mit Orgelpunkt fis, Takt 47-62 G-Dur gegen c-Moll mit Orgelpunkt $g$. In der Summe ergeben sich jedoch stets verschiedene Transpositionen einer Mixo b6 - Skala innerhalb der Heptatonia secunda.

Die tonale Organisation des Stückes ist aber noch weitaus rigoroser kalkuliert - ebenso stringent wie die polyphone Anlage. Betrachtet man nämlich die Abfolge der Orgelpunkte bzw. Zentraltöne, dann ergibt sich eine logisch konstruierte Reihe: $\underline{\mathrm{b}}, \mathrm{h}, \underline{\mathrm{d}}$, es, fis, g. (Bezugstöne der Refrainteile sind unterstrichen). Jedem Refrain schließt sich also ein Couplet an, dessen Zentralton einen Halbton höher liegt und jedem Couplet ein Refrain mit einem um eine kleine Terz höheren Bezugston, bis der Oktavraum diesem Prinzip gemäß vollständig durchschritten und der Ausgangston $b$ wieder erreicht ist. Bartók benutzt ein gemischtstufiges (und daher periodisches) Modell symmetrischer Oktavteilung, eine hexatonische Skala, die von dem Musiktheoretiker Zoltán Gárdonyi als alternierende Sechsstufigkeit (245ff), von Ernö Lendvai als Modell 1:3 bezeichnet wurde (Lendvai $35 \mathrm{ff}$ ). Sie ergibt sich durch lückenlose 
Aneinanderreihung eines identischen Intervallsegments aus Halbton und Anderthalbton (kleiner Terz), das sich dreimal im Oktavraum wiederholt. Die Periodik der Leiterstruktur lässt sich an der alternierenden Zahl der Halbtonschritte erkennen: $1-3,1-3,1-3$. Wie andere, vergleichbare Skalen zeigt auch die alternierende Sechsstufigkeit ein Distanzprinzip, d.h. es handelt sich um eine Tonhöhenordnung, die auf der Teilung der Oktave in gleiche oder alternierende Abstände und in diesem Sinne auf Periodizität beruht (Gárdonyi 156).

Distanzielle Phänomene gibt es in unterschiedlichen Ausprägungen. Bartók verwendet hier die alternierende Sechsstufigkeit zur Tonartendisposition im Sinne eines distanziellen Zirkels, als geschlossenen Kreis von Bezugspunkten innerhalb der Oktave. (Das Distanzprinzip findet sich in Bartóks Kompositionen erstmals nach seiner Forschungstätigkeit im BiskraGebiet 1913). Da die Heptatonia secunda Melodik und Harmonik von Subject und Reflection bestimmt, die Auswahl der einzelnen Transpositionen jedoch aus einem Modell symmetrischer Oktavteilung resultiert, liegt zweifelsfrei eine Vernetzung von zwei fundamentalen, im 20. Jhdt. neuen musikalischen Strukturprinzipien vor: Akustische und distanzielle Gestaltungsweisen durchdringen sich.

Bei soviel konstruktiver Logik bleibt die Frage nach der künstlerischen Intuition, nach einer besonderen kompositorischen Pointe des Stückes. Und tatsächlich ereignet sich Unerwartetes, Spannendes: Genau in dem Moment, als die distanzielle Achse komplett in einer Richtung abgeschritten ist und der letzte Refrain (Takt 63-73, Notenbeispiel 6) wieder den Orgelpunkt b als gemeinsame Spiegelachse aufnimmt, kommt es zu Variantenbildungen der Spiegelung. Als durchaus vergleichbares Kompositionsprinzip erweist sich die Veränderung der satztechnischen Faktur bei Wiederkehr einer Tonart in Johann Sebastian Bachs Fugen. Bartók versetzt die Pentachorde der rechten Hand in unterschiedliche Oktavlagen und verengt allmählich den ursprünglichen Quintambitus der linken Hand, zunächst auf die verminderte Quinte $e-b$ (Takt 63-66), dann auf die Quarte $f-b$ (Takt 66-69), schließlich auf die verminderte Quarte fis-b (Takt 70-73). Die deformierten Spiegelungen des Anfangspentachordes bedingen zwangsläufig auch Veränderungen der Binnenintervallik, so dass systemfremde Töne erscheinen ( $e, g, a$ sowie fis als enharmonische Variante von ges), die die bislang rein akustische Gesamttonalität in spannungsvoller Steigerung verfärben bzw. zu einer Chromatisierung des diatonischen Segments der Unterstimme führen. Ähnlich wie in Bachs Polyphonie bleibt jedoch die melodische Gestik trotz diastematischer Abweichungen 
grundsätzlich erhalten. Übrigens deutet eine geringfügige und scheinbar zufällige Modifikation der Spiegelung die späteren massiven Veränderungen schon vorher an: In Takt 44 und 46 fehlt der Ton cis.
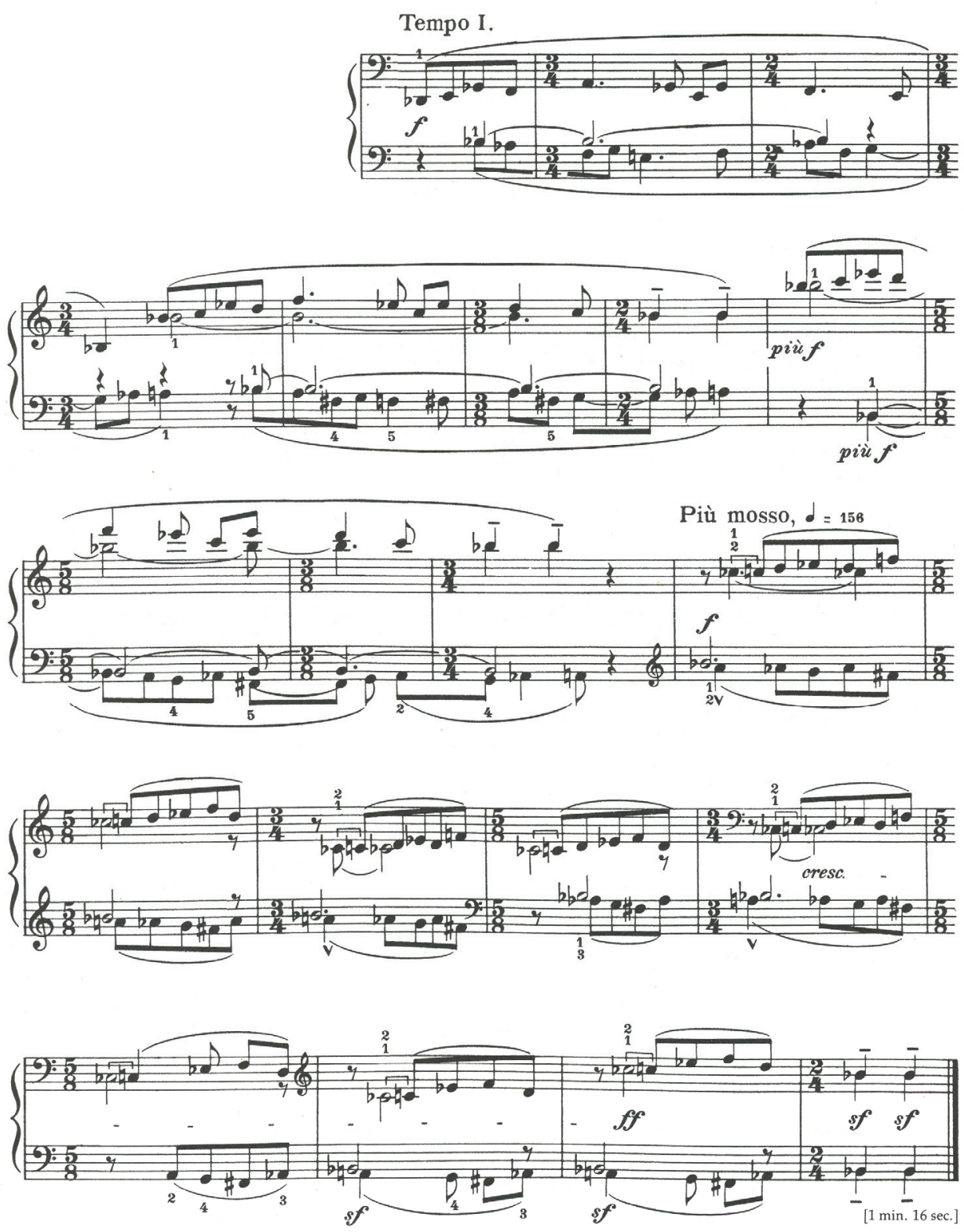

Notenbeispel 6. B. Bartók, Subject and Reflection (Tükrözödés), T. 63-82 
Der Schlussteil (Takt 74-82, Notenbeispiel 6) erzielt weitere Ausdruckssteigerung durch größere strukturelle Freiheit. Auch die dynamischen Angaben belegen die Kulmination. Gleichsam als höheres Prinzip überlagert intuitive Musikalität die Kalkulation: Die extrem strenge mathematische Gesetzmäßigkeit der Spiegelung wird noch weiter relativiert, die gemeinsame Spiegelachse entfällt. Das obere Pentachord hat den Ambitus der verminderten Quinte ces- $f$, das untere den der verminderten Quarte $f i s-b$. Der Tonvorrat umfasst zehn Töne, also fast das chromatische Total (nur des und $e$ fehlen). Die Unterstimme verläuft im chromatischen Tonraum, die Oberstimme im B-Dur-Bereich, ergänzt um ces. Trotz der starken Reibung kleiner und großer Sekunden bleibt der Orgelpunkt $b$ als Bezugston erkennbar. Deshalb liegt keine Atonikalität vor, sondern eher eine Art chromatisch erweiterter Tonalität, eine durch Reduktion nachweisbare latente Heptatonik (Engelmann 41; von der Nüll 58), sei sie diatonisch oder akustisch. So setzt sich auch gegen alle Trübung (...) immer wieder bis zum Schluss das (...) Leuchten der B-Dur-Melodie durch (Uhde 61). (Aus diesem Grund fehlt gerade der Ton des, da er die aus der Anfangsmotivik resultierende Durwirkung stören würde).

Bartóks Strukturalismus der Polyphonie und der tonalen Disposition in Subject and Reflection hat ästhetisch-philosophische Hintergründe: In einer pluralistischen Zeit der Auflösung und des Umbruchs mit stark divergierenden Entwicklungen können neue oder erneuerte Ordnungssysteme - gleich ob quasi natürlich oder konstruktiv - einen möglichen Ansatz zur Orientierung und Überbrückung von Differenzen bieten. (Auch Schönberg versuchte mit der Dodekaphonie ein derartiges Ordnungsprinzip zu gewährleisten). Allerdings erweist sich die vermeintlich gesicherte Stabilität und Ausgewogenheit jeder Ordnung stets als von der kompositorischen bzw. künstlerischen Inspiration relativierbar und auch nur als Station im historischen Prozess, als vorläufig, nicht endgültig.

\section{LITERATURVERZEICHNIS}

Bárdos, Lajos. „Die volksmusikalischen Tonleitern bei Liszt.” Franz Liszt-Beiträge ungarischer Autoren, hrsg. von Klara Hamburger, Corvina, 1978, S. 168-196.

Bárdos, Lajos. „Die volksmusikalischen Tonleitern bei Liszt.” Franz Liszt-Beiträge ungarischer Autoren, hrsg. von Klara Hamburger, Corvina, 1978.

Bárdos, Lajos. Harmine ira's. Zenemükiadó Vállalat, 1969.

Bárdos, Lajos. Modális harmóniák (Modale Harmonik). Kiadás éve, 1961.

Bartók, Béla. A magyar népdal (Das ungarische Volkslied). Rózsavölgyi és Társa, 1924.

Dahlhaus, Carl, u. Hans Heinrich Eggebrecht. Brockhaus-Riemann-Musiklexikon, Bd. 2, Schott, 1979. 
Dahlhaus, Carl. Die Musik des 19. Jahrhunderts. Laaber Akademische Verlagsanstalt, 1980.

Engelmann, Hans Ulrich. Béla Bartóks „Mikrokosmos”: Versuch einer Typologie „Neuer Musik.” Triltsch, 1953.

Gárdonyi, Zoltán. „Neue Ordnungsprinzipien der Tonhöhen in Liszts Frühwerken.” Franz Liszt Beiträge ungarischer Autoren, hrsg. von Klara Hamburger, Corvina, 1978, S. 226-273.

Gárdonyi, Zsolt, and Hubert Nordhoff. Harmonik. Möseler Verlag, 1990.

Haunschild, Frank. Die neue Harmonielehre, Bd. 1, AMA Verlag, 1988.

Lendvai, Ernö. Symmetrien in der Musik. Kodály Institut Kecskemét, 1995.

Lesznai, Lajos. Béla Bartók. Deutscher Verlag für Musik, 1967.

Lissa, Zofia. „Zur Genesis des „Prometheischen Akkords“ bei Alexandr Nikolajewitsch Skrjabin.” Musik des Ostens, Bd. 2, hrsg. von Johann-Gottfried-Herder-Forschungsstelle für Musikgeschichte, Bärenreiter, 1963, S. 170-183.

Persichetti, Vincent. Twentieth Century Harmony. W.W. Norton \& Co, 1961.

Somfai, László. „Bartók.” Die Musik in Geschichte und Gegenwart, Personenteil, Bd. 2, hrsg. von Ludwig Finscher, Bärenreiter Metzler, 1999.

Szabolcsi, Bence, Hrsg. Béla Bartók, Weg und Werk. Corvina 1957/Bärenreiter 1972.

Uhde, Jürgen. Bartóks Mikrokosmos: Spielanweisungen und Erläuterungen. Gustav Bosse Verlag, 1954.

von der Nüll, Edwin. Béla Bartók. Ein Beitrag zur Morphologie der neuen Musik. Mitteldeutsche Verlags-Aktien-Gesellschaft, 1930.

\section{KONSTRUKTYWIZM I INTUICJA. \\ ANALIZA UTWORU FORTEPIANOWEGO BÉLI BARTÓKA TÜKRÖZŐDÉS Z VI TOMU ZBIORU MIKROKOSMOS}

Streszczenie

Przedmiotem badań jest utwór fortepianowy Béli Bartóka: Tükrözödés z VI tomu zbioru Mikrokosmos. Autor analizując szczegółowo tę kompozycję, dowodzi, iż strukturalizm polifonii zaproponowany przez węgierskiego kompozytora i jej tonalne podłoże mają głębokie podłoże estetyczno-filozoficzne. Bartók poszedł własną drogą kompozytorską, na którą znaczny wpływ miało jego zainteresowanie folklorem. Na jego oryginalny język muzyczny złożyły się m.in. takie czynniki, jak: swobodne operowanie harmoniką, zwięzłość wypowiedzi muzycznej, właściwa melodiom i rytmom ludowym, a obca ekspresyjnej poetyce wykreowanej przez twórców XIX wieku, czy śmiałość w stosowaniu dysonansowych współbrzmień.

Słowa kluczowe: Béla Bartók; Tükrözödés; muzyka fortepianowa; muzyka XX wieku. 


\title{
KONSTRUKTIVISMUS UND INTUITION. \\ EINE ANALYSE VON BÉLA BARTÓKS KLAVIERSTÜCK SUBJECT AND REFLECTION AUS DEM MIKROKOSMOS
}

\begin{abstract}
Abstrakt
Das Forschungsthema dieses Artikels ist ein Klavierstück von Béla Bartók: Subject and Reflection (Tükrözödés) aus dem 6. Band des Mikrokosmos. Der Komponist beweist, dass der Strukturalismus der Polyphonie und seine tonale Grundlage einen tiefen ästhetisch-philosophischen Grund haben. Bartók ist stilistisch seinen eigenen Weg gegangen, der maßgeblich von seinem Interesse an der Folklore beeinflusst wurde. Seine musikalische Sprache ist durch mehrere Faktoren geprägt, u.a.: freier Umgang mit der Harmonik, Prägnanz des musikalischen Ausdrucks, typisch für Volksmelodien und Volksrhythmen, der expressiven Poetik der Komponisten des 19. Jahrhunderts fremd sind, oder Mut in der Verwendung dissonanter Akkorde.
\end{abstract}

Stichworte: Béla Bartók; Subject and Reflection; Klaviermusik; Musik des 20. Jahrhunderts.

\section{CONSTRUCTIVISM AND INTUITION. AN ANALYSIS OF BÉLA BARTÓK'S PIANO PIECE SUBJECT AND REFLECTION FROM MIKROKOSMOS VOL. VI}

\section{Summary}

The subject of research is the piano composition Subject and Reflection from Mikrokosmos (vol. VI) by Béla Bartók. Via a detailed analysis of this work, the author proves that the structuralism of polyphony proposed by the Hungarian composer, as well as its tonal background, has a deep aesthetic and philosophical background. Bartók followed his own creative path, which was significantly influenced by his interest in folklore. His original musical language was based on such factors as original harmonics, a conciseness of musical expression (typical of folk melodies and rhythms, and foreign to the expressive poetics created by the 19th century artists) and the courage to use dissonant chords.

Key words: Béla Bartók; Subject and Reflection; piano music; $20^{\text {th }}$ century music. 